\section{Jeder Schritt zählt - Sterblichkeit und objektiv erhobene Schrittzahl}

\section{Originalpublikation}

Saint-Maurice PF, Troiano RP, Bassett DR, et al. Association of Daily Step Count and Step Intensity With Mortality Among US Adults. Jama 2020; 323: 1151-1160 [1]

\section{Einleitung}

Die positive Wirkung von körperlicher Aktivität auf die vorzeitige Versterbewahrscheinlichkeit ist bereits intensiv untersucht. Die wissenschaftlichen Studien hierzu reichen bis in die 1950er-Jahre zurück [2]. In den meisten Fällen liegen den Rechnungen jedoch Aktivitätsdaten aus schriftlichen Befragungen der Probanden zugrunde. Die Ableitung von verlässlichen Dosis-Wirkungs-Beziehungen gestaltet sich daher etwas schwierig. Saint-Maurice und Kollegen [1] haben nun die Aktivitätsdaten von knapp 5.000 Personen der National Health and Nutrition Examination Survey (NHANES) Kohorte untersucht. Das Besondere: Die Aktivitätsdaten, d. h. die Schrittzahlen wurden objektiv anhand von Akzelerometern erfasst und in Zusammenhang mit Daten der Sterberegister gebracht. Das mittlere Alter der Kohorte lag zum Zeitpunkt der Baseline Erhebung bei 57 Jahren, der mittlere Follow-Up Zeitraum beträgt 10 Jahre.

Im Durchschnitt legten die Probanden 9.000 Schritte pro Tag zurück, wobei die Daten in mehrere Perzentile

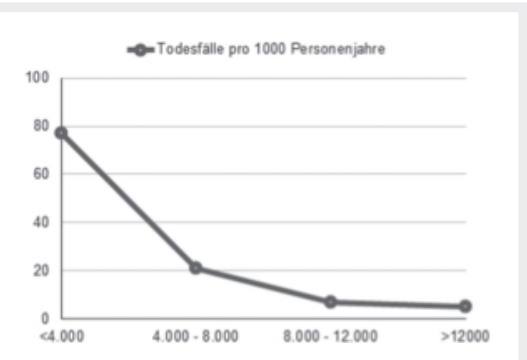

- Abb. 1Todesfälle pro 1.000 Personenjahre in Abhängigkeit der täglichen Schrittzahl. eingeteilt wurden, für welche jeweils die relative Versterbewahrscheinlichkeit innerhalb des Erhebungszeitraums erhoben wurde. Insgesamt wurden 1165 Todesfälle innerhalb dieses Zeitraums registriert.

Pro 1.000 Personenjahre wurden im inaktivsten Quartil 77 Todesfälle beobachtet. Dieses Verhältnis sinkt in den nächsten beiden Quartilen (4.000 bis 7.999 bzw. 8.000 bis 11.999 Schritte pro Tag) auf nur noch 21,4 bzw. 6,9 Todesfälle. Im aktivsten Quartil (>12.000 Schritte pro Tag) wurden lediglich 4,8 Todesfälle pro 1.000 Personenjahre beobachtet (Abb. 1). Im unadjustierten Modell entspricht dies einer Risikoreduktion von $90 \%$ seitens der aktivsten zur inaktivsten Gruppe. Im finalen statistischen Modell, d.h. nach Herausrechnen des Einflusses anderer relevanter Gesundheitsfaktoren zeigte sich eine Reduktion der vorzeitigen Versterbewahrscheinlichkeit von $65 \%$ in der aktivsten (>12.000 Schritte pro Tag) verglichen mit der inaktivsten Gruppe (<4.000 Schritte pro Tag). Durch die Adjustierung des Modells wird somit lediglich der isolierte Einfluss körperlicher Aktivität und potenzieller unbeobachteter, aber wahrscheinlich unbedeutender Variablen betrachtet. Auch für das Versterben durch eine kardiovaskuläre Erkrankung und Krebs werden Risikoreduktionen von $50 \%$ bzw. 33\% im aktivsten verglichen mit dem inaktivsten Quartil berichtet.

Die gefundenen Ergebnisse bestätigen andere vergleichbare Untersuchungen, wie beispielsweise die kürzlich publizierten Daten von Lee und Kollegen [3], die die Schrittzahlen von 17.500 Teilnehmerinnen der Women Health Study mit einem mittleren Alter von 72 Jahren $(S D=5,7)$ in Zusammenhang mit der Versterbewahrscheinlichkeit brachten. Auch hier beobachteten die Autoren den mit oben deckungsgleichen asymptotischen J-förmigen Zusammenhang. So konnte auch hier ein rasanter Risikoabfall von über $60 \%$ zwischen $<2.000$ und 7.500 Schritten verzeichnet werden. Danach trifft die Kurve auf ein Plateau bei einem verbleibenden Risiko von 35\% verglichen mit der inaktivsten Gruppe $\quad(<2.000$ Schritte proTag).

\section{Kommentar zur Studie}

Diese Daten bestätigen die bereits bekannte, enorme Bedeutung von körperlicher Aktivität auf das Gesamtüberleben anhand einer großen, repräsentativen USStichprobe und objektiv erhobener Aktivitätsdaten. Auch bestätigt sich der aus anderen Untersuchungen bekannte asymptotische Verlauf des Aktivitäts-Mortalitäts-Zusammenhangs, wonach bereits eine kleine inkrementelle Veränderung des Aktivitätsniveaus von „kaum” zu „ein wenig“ gewaltige Effekte mit sich bringt, die alle ferneren Steigerungen bei Weitem übertreffen. Die Daten zeigen außerdem, dass die generell empfohlenen 10.000 Schritte [4] zwar in den Optimalbereich (nahe des absoluten Minimums) fallen, allerdings nicht die höchste Effizienz (Knickpunkt der Kurve) zeigen, sondern diese eher bei 7.500 - 8.000 Schritten zu verorten ist. Welche Rolle anderen Beanspruchungsformen hierbei zukommt und ob diese einen additiven Mehrwert besitzen, bleibt in den genannten Studien unbeachtet. Es ist zu vermuten, dass ein regelmäßiges körperliches Training mit Kräftigungsanteilen einen zur hier betrachteten absoluten Schrittzahl zusätzlichen Gesundheitseffekt besitzt. So haben Ruiz et al. [5] einen starken Zusammenhang zwischen der Kraftfähigkeit und der Sterblichkeit beobachten können. Letzten Endes läuft es auf dieselbe Idee hinaus, wie sie in der DVGS-Bewegungspyramide [6] Verwendung findet. Die Basis eines gesunden Lebensstils bildet ein aktiver Alltag (>7.500 Schritte pro Tag), welcher mit intensiveren Belastungen und Kräftigungsübungen gespickt werden soll.

\section{ZUSATZINFO}

Dieser Journal Club Beitrag wurde bereits am 14.5.2020 publiziert unter https://dvgs.de/de/features/bl og/item/114-jeder-schritt-z\%C3\%A4h It-sterblichkeit-und-objektiv-erho bene-schrittzahl.html

\section{Korrespondenzadresse}

Maximilian Köppel

Deutscher Verband für Gesundheitssport und Sporttherapie e. V. maximilian.koeppel@outlook.de 